\title{
BIRBAL SAHNI INSTITUTE RADIOCARBON MEASUREMENTS I
}

\author{
G RAJAGOPALAN, VISHNU-MITTRE, and B SEKAR
}

Birbal Sahni Institute of Palaeobotany, Lucknow, India 226007

The Radiocarbon Dating Laboratory of the Institute was established in 1974 primarily to assist in the program of research undertaken by the Institute's Department of Quaternary Palynology (Rajagopalan and Vishnu-Mittre, 1977). The measurements carried out by the Laboratory up to December 1977 are presented in this clate list.

The specific activity of ${ }^{14} \mathrm{C}$ in samples is measured by counting methane synthesized from sample carbon (Agrawal et al, 1971) in an Oeschger-Houtermans gas proportional counter (Houtermans and Oeschger, 1958). The counter is filled to a pressure of $900 \mathrm{~mm}$ mercury. The background and net NBS oxalic acid counting rates were close to $2.45 \mathrm{cpm}$ and $10.25 \mathrm{cpm}$, respectively, throughout the measurement of the samples presented here. The experimental methods of methane synthesis are very similar to the ones described by Agrawal et al (1971). Samples are counted for 2500 minutes, initially, and a repeat counting for 1000 minutes is made after 30 days.

Dates are based on the value of 5570 years for the half-life of ${ }^{14} \mathrm{C}$ and $95 \%$ of the activity of NBS oxalic acid. The error on the date refers to $1 \sigma$ value which is calculated taking into account the counting statistics, uncertainty in the half-life and instability in the counting system (Kusumgar et al, 1963; Kusumgar, 1973). $\delta^{13} \mathrm{C}$ measurements have not been made on these samples.

We have carried out measurements of the specific activity of ${ }^{14} \mathrm{C}$ on a number of samples kindly supplied by D P Agrawal for cross-checking purposes. These samples are portions of well-dated charcoal samples from archaeologic sites in India. Table 1 presents the comparison on measurements made at the Birbal Sahni Institute and at the Tata Institute Lab, Bombay.

\section{ACKNOWLEDGMENTS}

The Laboratory received help and encouragement from T S Sadasivan, ex-Chairman, and K R Surange, Director, Birbal Sahni Institute of Palaeobotany in the initial phases of setting up. Their continued encouragement and interest in the growth of the laboratory is gratefully acknowledged. With deep gratitude we thank D P Agrawal for his help in construction of the laboratory and in supplying some equipment. We also thank D Lal, Director, Physical Research Laboratory, B V Sreekanton, Director, Tata Institute of Fundamental Research, and Rama for equipment and advice. We are grateful to $\mathrm{H}$ Oeschger for supplying the low-level counter.

Technical help from V S Panwar and P S Saluja are thankfully acknowledged.

The electronics units were constructed in the Electronics shop of the Physical Research Laboratory. One of us ( $G \mathrm{R})$ had the privilege of 
learning design details of the glass system at the Radiocarbon Lab in the Physical Research Laboratory. We are indebted to D P Agrawal, the staff of Radiocarbon Lab and the staff of the Electronics shop for help and facilities extended.

We are thankful to the Director of the Central Drug Research Institute, Lucknow for the supply of liquid air.

\section{SAMPLE DESCRIPTIONS QUATERNARY SAMPLES}

BS-2. Mandhata, Uttar Pradesh

$4380 \pm 130$

Carbonaceous sediments, depth 2 to $2.1 \mathrm{~m}$, from Horse Shore Lake, Mandhata $\left(25^{\circ} 40^{\prime} \mathrm{N}, 82^{\circ} \mathrm{E}\right)$, Dist Pratapgarh. Sample subm by H P Gupta, Birbal Sahni Inst Palaeobot (BSIP), Lucknow.

\section{Kashmir series}

Haigam Lake $\left(34^{\circ} 3^{\prime} \mathrm{N}, 74^{\circ} 29^{\prime} \mathrm{E}\right)$, Kashmir Valley. Clayey organic sediment samples coll 1963 by Vishnu-Mittre \& B D Sharma and subm by Vishnu-Mittre. Pollen diagram was previously pub (Vishnu-Mittre \& Sharma, 1966).

\section{BS-36. Carbonaceous clay}

$1640 \pm 115$

Depth 4 to $5 \mathrm{~m}$.

\section{BS-37. Carbonaceous clay}

Depth 5 to $6 \mathrm{~m}$.

Comment: pollen sequence was interpreted to date from Neolithic, ie, ca $5000 \mathrm{BP} .{ }^{14} \mathrm{C}$ dates are younger.

TABLE 1

Cross check samples

\begin{tabular}{|c|c|c|c|c|}
\hline Sample/site & $\begin{array}{l}\text { Birbal Sahni } \\
\text { Inst age (yr) }\end{array}$ & $\begin{array}{l}\text { Tata Inst } \\
\text { TF no. }\end{array}$ & age (yr) & Ref \\
\hline $\begin{array}{l}\text { Charcoal } \\
\text { Kalibangan }\end{array}$ & $3925 \pm 125$ & 163 & $3910 \pm 100$ & $\mathrm{R}, 1965, \mathrm{v} 7, \mathrm{p} 293$ \\
\hline $\begin{array}{l}\text { Charcoal } \\
\text { kalibangan }\end{array}$ & $3570 \pm 125$ & 152 & $3615 \pm 85$ & R, 1966, v 8, p 447 \\
\hline $\begin{array}{l}\text { Charcoal } \\
\text { Kalibangan }\end{array}$ & $3585 \pm 120$ & 149 & $3675 \pm 140$ & $\mathrm{R}, 1966, \mathrm{v} 8, \mathrm{p} 448$ \\
\hline $\begin{array}{l}\text { Charcoal } \\
\text { Kalibangan }\end{array}$ & $4010 \pm 165$ & 156 & $3740 \pm 105$ & $\mathrm{R}, 1966, \mathrm{v} 8, \mathrm{p} 448$ \\
\hline $\begin{array}{l}\text { Charcoal } \\
\text { Kayatha }\end{array}$ & $3490 \pm 125$ & 777 & $3625 \pm 95$ & $\mathrm{R}, 1969, \mathrm{v} 11, \mathrm{p} 503$ \\
\hline $\begin{array}{l}\text { Charcoal } \\
\text { Taklaghat }\end{array}$ & $2370 \pm 120$ & 784 & $2435 \pm 95$ & R, 1969, v 11, p 504 \\
\hline $\begin{array}{l}\text { Charcoal } \\
\text { Inamgaon }\end{array}$ & $2855 \pm 150$ & 923 & $2890 \pm 170$ & $\mathrm{R}, 1971, \mathrm{v} 13, \mathrm{p} 85$ \\
\hline $\begin{array}{l}\text { Charcoal } \\
\text { Nindwari }\end{array}$ & $3840 \pm 235$ & 862 & $3900 \pm 105$ & $\mathrm{R}, 1971, \mathrm{v} 13, \mathrm{p} 86$ \\
\hline $\begin{array}{l}\text { Charcoal } \\
\text { Paiyampalli }\end{array}$ & $1125 \pm 100$ & 829 & $985 \pm 105$ & $\mathrm{R}, 1971, \mathrm{v} 13, \mathrm{p} 87$ \\
\hline $\begin{array}{l}\text { Carbonized } \\
\text { kernals } \\
\text { Lena Athula }\end{array}$ & $7190 \pm 155$ & 1094 & $7640 \pm 110$ & $\mathrm{R}, 1975, \mathrm{v} 17, \mathrm{p} 220$ \\
\hline
\end{tabular}


Tsokar Lake $\left(33^{\circ} 20^{\prime} \mathrm{N}, 78^{\circ} \mathrm{E}\right)$, alt $4600 \mathrm{~m}$, Dist Ladakh. Samples coll by Geol Survey India, subm by Vishnu-Mittre dated for origin, history, vegetation, and climate of Tsokar I ake and region.

Samples from bore hole Core TP-6

BS-5. Carbonaceous clay

$11,830 \pm 500$

Depth 3m.

BS-9. Carbonaceous clay

$15,800 \pm 1110$

Depth $5.15 \mathrm{~m}$.

BS-11. Carbonaceous clay

$20,500 \pm 2000$

Depth $12 \mathrm{~m}$.

BS-17. Biogenic deposit

$30,600 \pm 1400$

Depth $21.85 \mathrm{~m}$.

\section{Samples from bore hole Core TSD-1}

BS-28. Biogenic deposit

$34,170 \pm 3370$

Depth 7.7 to $8.2 \mathrm{~m}$.

BS-29. Carbonaceous clay

$>40,000$

Depth 13.5 to $13.7 \mathrm{~m}$.

BS-30. Carbonaceous clay

$>41,000$

Depth $18.5 \mathrm{~m}$.

BS-31. Carbonaceous clay

$>15,000$

Depth 23.2 to $23.85 \mathrm{~m}$.

Comment: biogenic deposits from bore hole Cores TP-6 and TSD-1 (BS17 \& 28) indicate warm fluctuation within Weichselian glaciation. Pollen sequence is under preparation.

\section{Lahul series}

Batal $\left(32^{\circ} 14^{\prime} 30^{\prime \prime} \mathrm{N}, 77^{\circ} 33^{\prime} 40^{\prime \prime} \mathrm{E}\right)$, Lahul Valley, Dist Lahul \& Spiti. Samples coll from trial trench along river bed and subm by A Bhattacharya, BSIP. Samples date pollen sequence.

BS-54. Varved silty clay

$495 \pm 90$

Depth 7 to $17 \mathrm{~cm}$.

BS-60. Varved silty clay

$1370 \pm 135$

Depth 60 to $97 \mathrm{~cm}$.

\section{Rajasthan series}

Samples from trial trenches from lakes in Rajasthan desert coll and subm by A K Saxena, BSIP to date origin and history of these lakes and of vegetation and climate of Rajasthan desert.

Didwana Salt Lake $\left(27^{\circ} 30^{\prime} \mathrm{N}, 74^{\circ} 30^{\prime} \mathrm{E}\right)$, Dist Nagaur. 
BS-24. Laminated dark elay

$1960 \pm 160$

Depth 40 to $75 \mathrm{~cm}$.

BS-26. Laminated dark clay

$3725 \pm 125$

Depth 130 to $135 \mathrm{~cm}$.

BS-34. Laminated dark clay

Depth 230 to $235 \mathrm{~cm}$.

$6110 \pm 125$

BS-35. Laminated dark clay

Depth 290 to $295 \mathrm{~cm}$.

$7835 \pm 165$

Comment: pollen sequence from this lake pub earlier (Singh et al, 1974) had a single date (WIS-415-2970 \pm 65 вP) at depth $125 \mathrm{~cm}$. Present profile has consistent dates; bottom date agrees with estimates made by Singh et al (1974). Immigration of Calligonum polygonoides, a psammophytic sp, from extreme $\mathrm{W}$ of desert into vicinity of Didwana as observed in pollen diagram (Singh et al, 1974) is dated by BS-34 to ca $6000 \mathrm{BP}$. Pollen analysis of present profile is in progress.

Kanod $\left(29^{\circ} \mathrm{N}, 71^{\circ} \mathrm{E}\right)$, Dist Jaisalmer.

BS-50. Sandy clay

Depth $50 \mathrm{~cm}$.

$8700 \pm 200$

BS-51. Sandy elay

Depth $120 \mathrm{~cm}$.

$9570 \pm 160$

Comment: deposits of Kanod playa are dated to 10,000 $\mathrm{BP}$, same as some of salt lakes in $\mathrm{N}$ of Rajasthan desert. Sedges dominated grasses in vicinity of this playa until 8700 BP. Thereafter, grasses dominated. Pollen grains of thorn forest elements are scarce.

Pushkar lake (262 $\left.29^{\prime} \mathrm{N}, 74^{\circ} 33^{\prime} 50^{\prime \prime} \mathrm{E}\right)$, Dist Ajmer.

BS-12. Sandy clay

Depth 110 to $115 \mathrm{~cm}$.

Modern

BS-13. Sandy clay

Depth 130 to $135 \mathrm{~cm}$.

$1035 \pm 110$

Comment: beginning of Anogeissus forest with low frequencies of Calligonum and abundance of sedges is dated by BS-13. This is in contrast to changes observed at nearly same depth in Pushkar lake diagram pub by Singh et al (1974).

Budh Pushkar Lake (262 $29^{\prime}$ N, $74^{\circ} 33^{\prime} 50^{\prime \prime}$ E), Dist Ajmer.

BS-4. Sandy clay

Depth 20 to $25 \mathrm{~cm}$.

Modern

BS-6. Sandy clay

Depth 105 to $110 \mathrm{~cm}$.

$425 \pm 80$

Commenl: BS-6 dates occurrence of grassland-chenopod savamnah in vicinity of lake. 


\section{Nilgiri series}

Colgrain $\left(11^{\circ} 35^{\prime} \mathrm{N}, 76^{\circ} 52^{\prime} \mathrm{E}\right)$, Dist Nilgiris. Samples from bore holes in peat deposits, subm by $\mathrm{K}$ Prasad, BSIP to date pollen diagram.

BS-19. Peaty clay

$7840 \pm 125$

Depth 20 to $50 \mathrm{~cm}$.

BS-20. Clay

$27,450 \pm 1000$

Depth 70 to $100 \mathrm{~cm}$.

BS-21. Peaty clay

$21,350 \pm 450$

Depth 120 to $150 \mathrm{~cm}$.

BS-23. Peaty elay

$14,500 \pm 930$

Depth 220 to $250 \mathrm{~cm}$.

Comment: middle 2 dates are highly inconsistent. BS-23 dates profile from late Weichselian. Pollen diagram reveals that ca 15,000 yr ago a grassland with Impatiens occurred around Colgrain and Shola forest was established here ca 8000 yr ago (Vishnu-Nittre \& Gupta, 1971).

Upper Bhawani $\left(11^{\circ} 21^{\prime} \mathrm{N}, 76^{\circ} 45^{\prime} \mathrm{E}\right)$, Dist Nilgiris. Samples from bore hole in peat deposits coll and subm by H P Gupta. Samples date pollen diagram.

BS-52. Peaty clay

$5690 \pm 110$

Depth $1.4 \mathrm{~m}$.

BS-53. Peaty clay

$18,540 \pm 290$

Depth $2.15 \mathrm{~m}$.

Comment: hiatus in stratigraphy seems involved. Pollen analysis of profile is in progress.

GEOLOGIC SAMPLES

BS-7. Nainital flats, Uttar Pradesh

$1470 \pm 100$

Wood assoc with landslide debris recovered by drilling at depth $61 \mathrm{~m}$ from Nainital Flats $\left(29^{\circ} 23^{\prime} 30^{\prime \prime}\right.$ N, $79^{\circ} 27^{\prime} 30^{\prime \prime}$ E), Dist Nainital. Samples subm by Geol Survey of India.

BS-8. Mothranwala Swamp, Uttar Pradesh

$585 \pm 140$

Clay from Mothranwala swamp $\left(30^{\circ} 15^{\prime} \mathrm{N}, 78^{\circ} 2^{\prime} \mathrm{E}\right)$ alt $680 \mathrm{~m}$ Dist Dehra Dun. Sample, depth 70 to $90 \mathrm{~cm}$, subm by B S Venkatachala, Indian Inst Petroleum.

BS-18. Corubathan, Bengal

$250 \pm 115$

Charcoal fragments from excavation at Corubathan $\left(27^{\circ} 6^{\prime} 6^{\prime \prime} \mathrm{N}\right.$, $88^{\circ} 48^{\prime} 53^{\prime \prime}$ E), Dist Darjeeling. Excavated across exposed terrace. Subm by $S$ Ghosh, Geol Survey India.

BS-33. Vettikod, Kerala

Wood fragment removed from stump in natural exposure, pit depth $1.5 \mathrm{~m}$ at Vettikod $\left(9^{\circ} 10^{\prime} 30^{\prime \prime} \mathrm{N}, 76^{\circ} 35^{\prime} 15^{\prime \prime} \mathrm{E}\right)$, Dist Allepey. 
BS-32. Vettikod, Kerala

I.ignite from natural, exposure, pit depth $1.5 \mathrm{~m}$ at Vettikorl $\left(9^{\circ} 10^{\prime}\right.$ $\left.30^{\prime \prime} \mathrm{N}, 76^{\circ} 35^{\prime} 15^{\prime \prime} \mathrm{E}\right)$, Dist Allepey.

\section{BS-27. Kutheravattam, Kerala}

Lignite from well dug in Kutheravattam $\left(9^{\circ} 11^{\prime} \mathrm{N}, 76^{\circ} 36^{\prime} 40^{\prime \prime} \mathrm{E}\right)$, Dist Allepey. Sample from depth 8m. Samples BS-39, 32 and 27 subm by Dir Geochronol Div, Geol Survey India to date occurrence of lignite in Kerala. Comment: lignite deposits in Kerala are believed by geologists to be of Late Tertiary age (Wadlia, 1961). It is interesting to find that BS-27 is clated to late Quaternary.

\section{BS-47. Rajdanda, Bihar}

$36,560 \pm 2535$

Carbonized wood from natural exposures, $1.5 \mathrm{~m}$ below surface, along

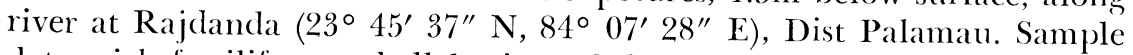
dates rich fossiliferous shell horizon. Subm by V P Misra, Geol Survey India.

\section{BS-48. Khetri, Rajasthan}

$$
140 \pm 90
$$

Gray-colored fire wood stacked at head of Madan (Khetri) copper mine $\left(28^{\circ} \mathrm{N}, 75^{\circ} 47^{\prime} \mathrm{E}\right)$, Dist Jhunjhunu. Date of copper mining in area is too recent; it was known to be much earlier from other evidence.

\section{BS-15. Tilapat, Haryana}

\section{ARGHAEOLOGIC SAMPLES}

Decaying timber (Adansonia digitata) from exposed stump) at Tilapat $\left(28^{\circ} 30^{\prime} \mathrm{N}, 77^{\circ} 20^{\prime} \mathrm{E}\right)$, Dist Gurgaon. Felling date of tree is unknown but is believed to have been planted during Mahabaratha period. Subm by $\mathrm{K}$ M Vaid, Forest Research Inst, India. Comment: younger than expected.

\section{BS-38. Appukullu, Madras}

Charcoal from APKL-1. Systematic excavation at Appukullu $\left(12^{\circ}\right.$ $\left.52^{\prime} \mathrm{N}, 78^{\circ} 59^{\prime} 2^{\prime \prime} \mathrm{E}\right)$, Dist N Arcot. Subm by K V Raman, Univ Madras. Dated for sequence and chronology of Neolithic and Megalithic cultures of Tamil Nadu.

\section{BS-39. Lumbini, Nepal}

Charcoal, Sample 3, depth 2m, from Lumbini $\left(27^{\circ} 20^{\prime} \mathrm{N}, 83^{\circ} 30^{\prime} \mathrm{E}\right)$, Dist Taulihawa, believed to be birth place of Lord Buddha, Nepal. Systematic excavation by Dir Archaeol, HMG, Nepal, and Archáeol Adviser, Indian Cooperation Mission, Nepal. Subm by Vishnu-Mittre. Date is close to archaeol estimate.

\section{BS-42. Marakadola, Assam}

$$
660 \pm 95
$$

Charcoal from Trench 3, systematic excavation, Marakadola $\left(26^{\circ} \mathrm{N}\right.$, $\left.91^{\circ} 48^{\prime} \mathrm{E}\right)$, Dist Kamrup. Subm by S N Rao, North Eastern Hill Univ, Shillong. 
Carbonized rice coll from a pit, depth $40 \mathrm{~cm}$, at Dazelling $\left(27^{\circ} 30^{\prime} \mathrm{N}\right.$, $\left.92^{\circ} 20^{\prime} \mathrm{E}\right)$, Dist Kameng. Subm by S K Dutta, Dibrugarh Univ, Assam.

BS-40. Off-Bombay Coast

OCEANOGRAPHIC SAMPLES

Sediment core coll by R V Oceanographer, Off-Bombay Coast $\left(20^{\circ}\right.$ $\left.10^{\prime} \mathrm{N}, 70^{\circ} 26^{\prime} 59^{\prime \prime} \mathrm{E}\right)$. Top sample of Core 4. Subm by M G A P Shetty, Natl Inst Oceanog (NIO), Goa.

BS-45. Off-Bombay Coast

$20,940 \pm 450$

Sediment core coll by RV Oceanographer, Off-Bombay Coast (18 $\left.35^{\prime} 12^{\prime \prime} \mathrm{N}, 69^{\circ} 17^{\prime} 12^{\prime \prime} \mathrm{E}\right)$. Bottom sample, No. 7, of Core 2. Subm by M G A P Shetty, NIO, Goa.

\section{GEOPHYSICAL SAMPLES}

\section{Minicoy series}

Dead corals from natural exposure near Light House, Minicoy $\left(8^{\circ} 0^{\prime}\right.$ $\left.8^{\prime \prime} \mathrm{N}, 73^{\circ} \mathrm{E}\right)$. Sample to date storm beaches and formation of islands. Subm by H N Siddiquie, NIO, Goa.

BS-56.

Modern

BS-57.

$205 \pm 105$

REFIRENCFS

Iorawal, D P, Gupta, S K and Kusumgar, Shecla, 1971, Tata Institute radiocarbon date list IX: Radiocarbon, v 13, p 442-449.

Houtermans, F G and Oeschger, H, 1958, Proportional zählrohr zur Messung Schwacher Activitaten Weicher $\beta$ Stralung: Helvetica Phys Acta, v 31, p 117-126.

Kusumgar, Sheela, 1973, Problems of errors in radiocarbon dating, in: Radiocarbon and Indian archacology, D P Agrawal and $\Lambda$ Ghosh (eds), Tata Institute of Fundamental Rescarch, Bombay.

Kusumgar, S, Lal, D) and Sharma V K, 1963, Radiocarbon dating techniques: Indian Acall Sci Proc sec A, v 58, no. 3, p 125-141.

Rajagopalan, $G$ and Vishnu-Mittre, 1977. Radiocarbon dating programme at the Birbal Sahni Institute of Palacobotany, Iucknow: Internatl conf low radioactivity measurements and applications, Proc. High Tatras, 6-10 Oct 1975, p 335-340.

Singh, G, Joshi, R I), Chopra, S K and Singh, A B, 1974, Late Quaternary history of vegetation and climate of Rajasthan desert, India: Royal Soc [London] Philos Trians, v 267B (889), p 467-501.

Vishum- Mitte and Sharma, B D, 1966, Studies on postglacial vegetational history from the Kashmir Valley-1, Haigam Lake: Palacobotanist, v 15 (1 \& 2), p 185 -2 12 .

vishnu-Mitte and Gupta, H P, 1971, ()rigin of Sholat forests in the Nilgiris, South India: Palacobotanist, v 19, no. 1, p $110-114$

Wadia, I) N, 1961, Geology of India, Macmillan, I London, p 459-461. 